\title{
ASSESSMENT AND REQUIREMENTS OF NUCLEAR REACTION DATABASES FOR GCR TRANSPORT IN THE ATMOSPHERE AND STRUCTURES
}

3

\author{
F. A. Cucinotta*, J. W. Wilson*, J. L. Shinn* and R. K. Tripathi** \\ *NASA, Langley Research Center, Hampton VA 23681-0001, U.S.A. \\ **Hampton University, Hampton VA 23662, U.S.A.
}

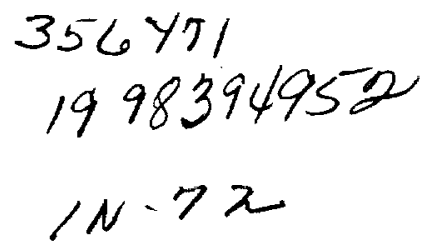

\begin{abstract}
The transport properties of galactic cosmic rays (GCR) in the atmosphere, material structures, and human body (self-shielding) are of interest in risk assessment for supersonic and subsonic aircraft and for space travel in low-Earth orbit and on interplanetary missions. Nuclear reactions, such as knockout and fragmentation, present large modifications of particle type and energies of the galactic cosmic rays in penetrating materials. We make an assessment of the current nuclear reaction models and improvements in these model for developing required transport code data bases. A new fragmentation data base (QMSFRG) based on microscopic models is compared to the NUCFRG2 model and implications for shield assessment made using the HZETRN radiation transport code. For deep penetration problems, the build-up of light particles, such as nucleons, light clusters and mesons from nuclear reactions in conjunction with the absorption of the heavy ions, leads to the dominance of the charge $Z=0,1$, and 2 hadrons in the exposures at large penetration depths. Light particles are produced through nuclear or cluster knockout and in evaporation events with characteristically distinct spectra which play unique roles in the build-up of secondary radiation's in shielding. We describe models of light particle production in nucleon and heavy ion induced reactions and make an assessment of the importance of light particle multiplicity and spectral parameters in these exposures.

Published by Elsevier Science Ltd on behalf of COSPAR.
\end{abstract}

\section{INTRODUCTION}

The description of the transport properties of galactic cosmic rays (GCR) is of interest for reasons as diverse as the understanding of the inter-stellar composition and the age of the universe to assessing radiation safety for humans seeking to embark into space for long-term orbits or interplanetary travel. The description of the passage of the GCR through matter is through the Boltzmann equation which takes into account the atomic and nuclear processes that lead to energy loss, absorption and production of new particles in interactions with shielding materials (Wilson et al., 1991). The accurate solution of this equation relies on accurate numerical methods and atomic and nuclear data bases. Research at Langley Research Center since the 1970's has focused on each of these aspects with application to the NASA Radiation Health Program as the chief focus. The development of one-dimensional transport codes using the straight-ahead approximation has been described by Wilson et al. (1991) and are in an advanced stage of development. Recent spaceflight measurements of light ion charged particle spectrum by Badhwar et al. (1994) highlight the effects of secondary nuclear reactions where fluxes of $n, d, t, h$, and $\alpha$ ions below $100 \mathrm{MeV} / \mathrm{u}$ showed a combined flux close to the primary and secondary proton flux. In low earth orbit, the geomagnetic effects prevent primaries from this energy regime and the fluxes seen are attributed solely to secondary fragments produced in the shielding. The nuclear databases breakdown into several areas including the heavy ion absorption and fragmentation cross sections, the proton, neutron and other light ion production multiplicity's and energy spectrum, and the meson production multiplicity's and energy spectra. Each of these areas can be further separated based on reaction partners as the dynamics of reactions change with kinetic energy and mass of the reaction partners. 
Continued emphasis on the description of heavy ion fragmentation from both a theoretical and experimental viewpoint is needed for improving and validating data bases. In Figure 1 a $x^{2}$ comparison of the current database NUCFRG2 (Wilson et al., 1994) is made with existing measurements. Also in Figure 1 is a comparison of different measurements with identical ion combinations made with nearly the same beam energies. This comparison shows that any improvements in the theoretical modelling of heavy ion fragmentation cross sections is presently limited by systematic errors in the existing measurements. New measurements with relativistic Fe beams by Zeitlin et al. (1996) should help to reduce this systematic differences in previous measurements. Further measurements with the important GCR components and with several target materials of interest are also warranted.

The theoretical description of fragmentation reactions is generally made in the abrasion-ablation model. There have been several formulations of these models using frameworks of classical dynamics (Bowman et al., 1973, Wilson et al., 1987) or quantum models based on the Glauber theory (Hufner et al., 1975),
optical potential models (Townsend et al., 1986), or through considerations of quantum transition using the high energy approximate multiple scattering series (Cucinotta and Dubey, 1994). In this paper we consider a new nuclear data base for GCR transport based on the quantum model, QMSFRG, and make comparisons using the HZETRN transport model to the data base NUCFRG2 which is based on the classical dynamics approach. We also will make some comparisons with the HZETRN code of the importance of light particle production such as neutrons, proton, deuterons, and alpha particles. The build-up of these ions in shielding materials leads to their dominance in astronaut exposures behind heavily shielded compartments (Cucinotta et al., 1995a).

\section{EVOLUTION OF FRAGMENTATION DATA BASES}

In the abrasion-ablation models, the abrasion step is the description of the initial overlap of the projectile and target nuclei and the removal of nucleons or mass in the overlap. The remains of the projectile (or target) in abrasion is denoted the pre-fragment and is expected to be in an excited state. The pre-fragment decays to a stable nucleus through particle or gamma-ray emission to form the final fragment. The NUCFRG model (Wilson et al., 1987) evolved from the liquid drop abrasion-ablation model of Bowman et al. (1973) with several improvements including the description of the excitation energies after abrasion from frictional

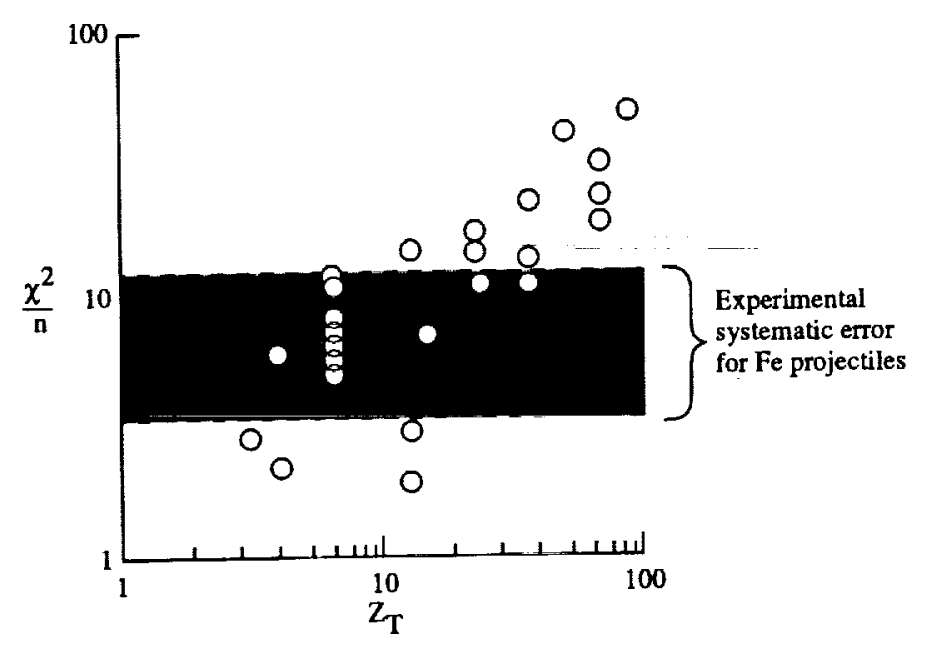

Fig. 1. A statistical comparison of the NUCFRG2 data base to experiments with Fe beams on several targets. Also shown is statistical comparison between measurements with Fe beams on several targets with nearly identical beam energies used. 
interactions, and application of a modified form of the Rudstam model (1966) for the description of the charge distribution of the final fragments. The NUCFRG model was the first to be used to generate a data base for GCR radiation transport codes, although parametic fits to experiments were used earlier. Early versions of the HZETRN code used energy independent cross sections with fragmentation parameters set at $2 \mathrm{GeV} / \mathrm{u}$ and considered only elemental distributions in the transport solutions. This was followed by studies of both the mass distribution of the ion fields and the energy dependence of the cross sections. At the same time the light ions $d, t, h$, and $\alpha$ were separated from the heavy ion transport algorithms due to their broader energy spectrum and distinct mechanisms for nuclear interactions were direct interactions dominate (Cucinotta, 1993).

A modified form of the NUCFRG model (NUCFRG2) (Wilson et al., 1994) considers Coulomb trajectories (important below $50 \mathrm{MeV} / \mathrm{u}$ ), energy dependent mean free path inside the nucleus, and ad-hoc corrections to the excitation energies. The NUCFRG 2 data base also renormalizes the cross sections for fragments with mass number greater than 4 to match values for the nuclear absorption cross section. The energy dependent corrections to the HZETRN code and the NUCFRG2 model were shown to alter substantially the prediction of both attenuation and flux of heavy ion behind spacecraft shielding (Shinn et al., 1993). Studies of the convergence of the mass spectra of ions indicated about 60 ions are necessary for GCR transport while about 150 ions are required for detailed studies with laboratory $\mathrm{Fe}$ beams (Kim et al., 1994). Further improvements in the NUCFRG model are expected to be limited due to the need to describe quantum effects such as the diffusness of the nuclear surface and nuclear structure and clustering, as well as improvements in the treatment of nuclear structure in the ablation stage where the nuclear level density of nuclei with mass below $\mathrm{A}<60$ are expected to be strongly effected by shell effects for excitation energies below $100 \mathrm{MeV}$. A quantum approach for generating a GCR data base is described next.

\section{QMSFRG: QUANTUM APPROACH TO FRAGMENTATION DATABASE}

The nuclear interaction is confined to short-distances and is highly singular. Consideration of quantum models for the formulation of interaction cross sections must then be based on the multiple scattering series (QMS). For high energy reactions, the eikonal approximation which assumes that the scattering is peaked at forward angles is used and leads to a great simplification in the summation of the multiple scattering series. Early work with the heavy ion QMS introduced an optical potential to describe the nuclear absorption cross section. A formalism for the abrasion cross section was derived by Hufner et al. (1975) using the Glauber form of the QMS and this was reconsidered by Townsend et al. (1986) using an optical model. These earlier works used closure approximations to integrate over the coordinates of abraded nucleons and the prefragment states to obtain the total strength for the abrasion of a $n$ nucleons in a fragmentation reaction. We have recently have reformulated the abrasion model to consider the quantum transitions of the pre-fragment (Cucinotta and Dubey, 1994). This approach allows nuclear structure and nuclear clustering effects to be considered in the model. For the abrasion of a large number of nucleons the use of a purely quantum approach becomes impractical and statistical methods for analysing the pre-fragment excitation energy distribution are being developed in order to introduce a continuous distribution of pre-fragment excitation energies.

The NUCFRG2 model considers only a minimal model of the evaporation cascade in the ablation stage with the removal of one mass unit for every $10 \mathrm{MeV}$ excitation and applies a phenomenological charge distribution to the predicted mass distribution of the model. The approach of Hufner et al. (1975) and Townsend et al. (1986) has been to use a Monte-Carlo simulation of the evaporation cascade. We have developed a deterministic approach to the evaporation cascade based on the Master equation for the de-exciation of a prefragment (Cucinotta and Wilson, 1996). An iterative solution to the Master equation has been developed which is convergent for excitation energies up to about $150 \mathrm{MeV}$ in the mass range $A<60$ and for all excitation energies for pre-fragments with $\mathrm{A}<16$. For higher excitation energies and large pre-fragment mass an approximate solution to the Master equation is used (Campi and Hufner, 1981). The model considers detailed level density appropriate for the mass range of interest for cosmic ray studies where shell effects are important and uses experimental determined branching ratios for decay of lighter nuclei $\mathrm{A}<12$ where the statistical evaporation model is expected to be less accurate. 
In Figure 2 we compare the QMSFRG model to the NUCFRG2 model and experimental data for high energy $\mathrm{Mg}$ and Fe beams. The QMSFRG model leads to better agreement with the data and is able to describe the odd-even effect in the elemental distribution as seen in the data. The cross sections for $p$ and $n$ production are very close in the model which indicates that these light ions are produced mostly from the abrasion stage of the reaction where the models give similar results for the total absorption. In Figure 3 we show comparisons of the models for the macroscopic cross sections for the mass distribution for Si and $\mathrm{Ca}$ beams. Clearly, there are substantial differences in the predictions of the models. The differences are greatest for few nucleon removal and for immediate mass projectiles and the importance of such differences for GCR transport is next evaluated.

\section{GCR MASS DISTRIBUTIONS: QMSFRG VS. NUCFRG2}

We next discuss comparison of the mass distribution of the GCR behind spacecraft shielding calculated using the HZETRN computer code with the 1977 solar minimum input spectra and assuming spacecraft

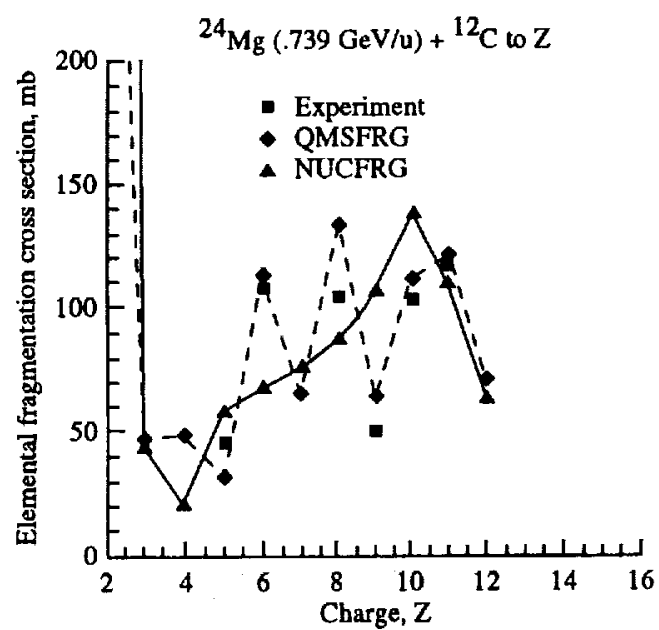

Fig. 2a. Comparison of QMSFRG, NUCFRG2, and experiment for elemental production cross sections for $\mathrm{Mg}$ fragmentation on $\mathrm{C}$ targets at $0.739 \mathrm{GeV} / \mathrm{u}$.

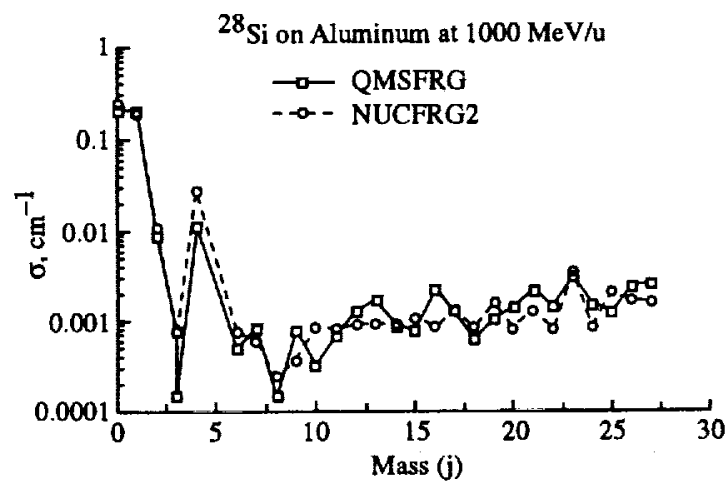

Fig. 3a. Comparison of mass distribution of fragments between QMSFRG and NUCFRG2 for fragmentation of $\mathrm{Si}$ on $\mathrm{Al}$ at $1 \mathrm{Ge} \mathrm{V/u}$.

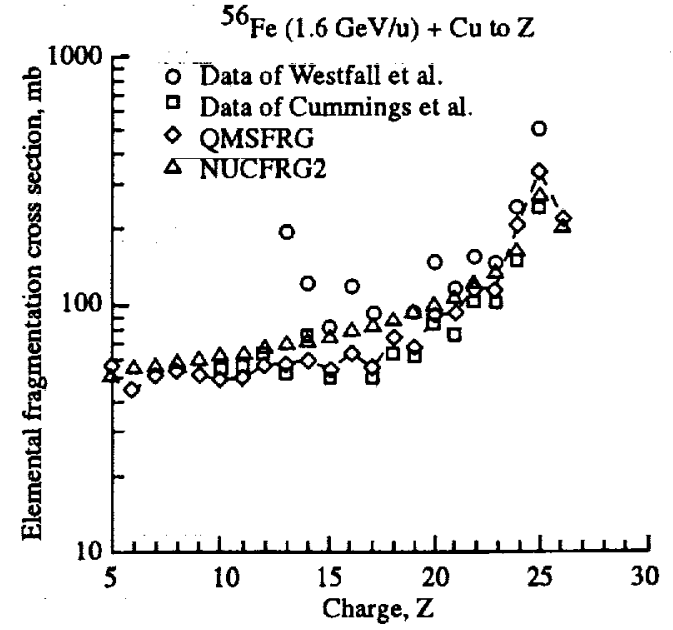

Fig. 2b. Same as Fig. 2a for $\mathrm{Fe}$ on $\mathrm{Cu}$ at $1.6 \mathrm{GeV} / \mathrm{u}$.

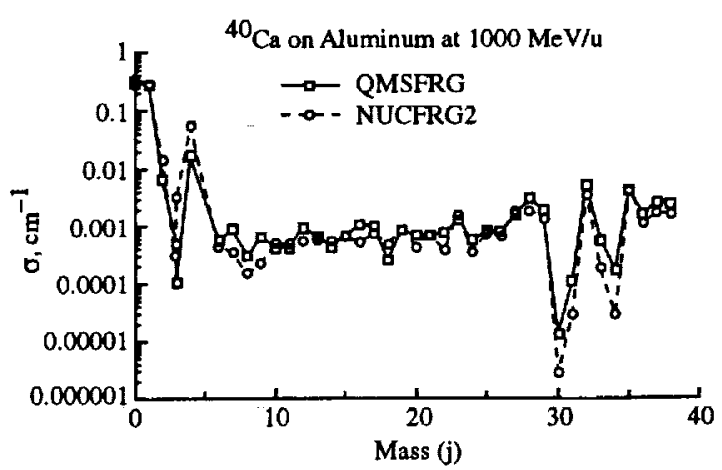

Fig. 3b. Same as Fig. 3a for $\mathrm{Ca}$ on $\mathrm{Al}$ at $1 \mathrm{GeV} / \mathrm{u}$. 
shielding of aluminium. Comparisons with other materials will be discussed elsewhere. We note that the NUCFRG2 code compiles the complete fragment distribution for the fragmentation of nuclei with masses between 6 and 58 and for 7 projectile energies in only several minutes on a VAX 4000 series computer or recent model personal computer. In contrast the QMSFRG model has a cpu time of about 24 hours on a VAX 4000. This is a reasonable CPU time for data base construction which is made once and stored for each material type for input into GCR transport codes.

In Figures 4 we show the ratio of the mass distributions for the two databases at depths of 3,10 , and $20 \mathrm{~g} / \mathrm{cm}^{2}$ of aluminium. In the HZETRN code the mass index $j$ corresponds to the most stable mass ion for $j>6$ and in Figure 4 the neutrons are shown at $j=0$ and the $\mathrm{H}$ and $\mathrm{He}$ isotopes are represented at $j=1$ to 5 . These comparisons show that for ions abundant in the primary GCR spectrum that very similar predictions are made for the 2 data bases with the ratios within the range from 0.95 to 1.05 . However, for the GCR fluxes where the primary fluxes are small the models give larger differences, in some cases the ratios show differences approaching a factor of 10. This comes about for several reasons; including the importance of atomic interactions on the energy loss of heavy ions which dominates the transport properties of the lower energy GCR components. Also, the NUCFRG2 model is renormalized to parameterized absorption cross sections that are nearly identical to predictions of the QMS model. Finally, the production cross sections are most distinct for fragments off the mass stability curve. These comparisons suggest further experimental and theoretical studies which detail the elemental and mass distributions will aid in the development of models. The near convergence of these 2 distinct data base generators for the most abundant heavy ion components is quite encouraging and future work with other shielding material and with laboratory beams where the energy dependence of the cross sections can be isolated should be persued.

\section{LIGHT ION PRODUCTION}

As shown previously, the light ion projectiles and secondaries dominate the exposures as large shielding depths are penetrated (Cucinotta et al., 1995a). The selection of material composition will have a large bearing on the transport properties of the light ions, including neutrons, hydrogen and helium isotopes. This is illustrated in Figure 5 where we show the total flux of these ions versus shielding depth in liquid hydrogen, water, and aluminium. Hydrogen shielding is optimal due to the absence of target fragmentation which has been shown to lead to a substantial build-up of light ions (Cucinotta et al., 1995a). Also shown in Figure 5 is the contribution to light particle production from $\alpha$ and HI's. Their relative contribution to $n, d$, and $\alpha$ production increases with decreasing shielding mass with about $30 \%$ of light particle production due to $\alpha$ and HI induced reactions in a water shield. The $\alpha$ and HI's will contribute about equally to these secondary ions (Cucinotta 1993). In the atmosphere, values close to those shown here for water should be expected.

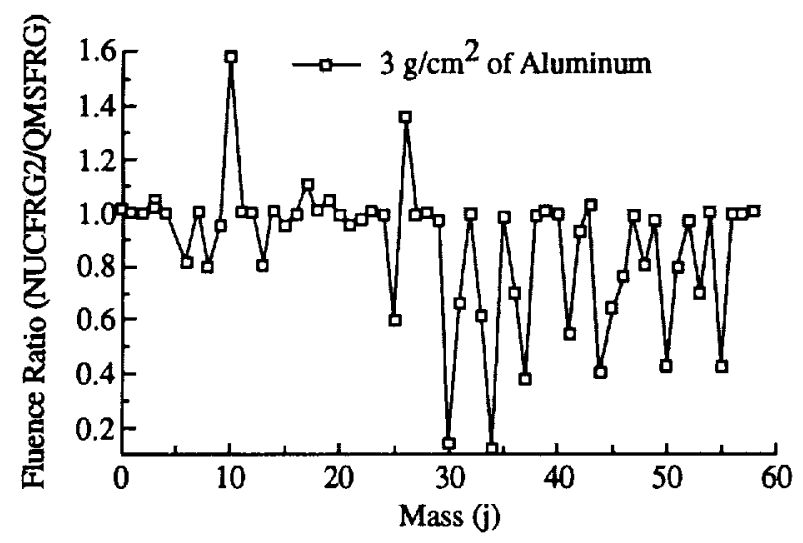

Fig. 4a. Ratio of fluences of ions of mass $\mathrm{j}$ calculated with HZETRN using NUCFRG2 data base to ones using QMSFRG database at $3 \mathrm{~g} / \mathrm{cm}^{2}$ of Al shielding. 


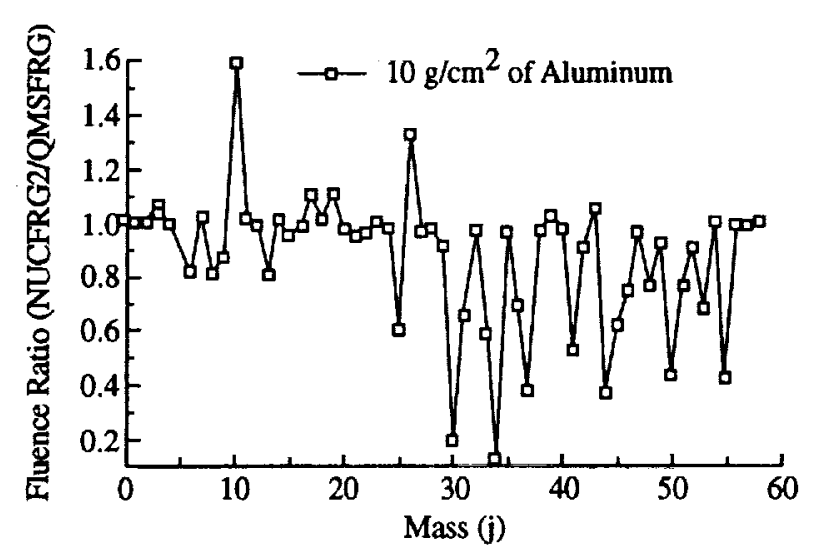

Fig. $4 b$. Same as $4 \mathrm{a}$ for $10 \mathrm{~g} / \mathrm{cm}^{2}$.

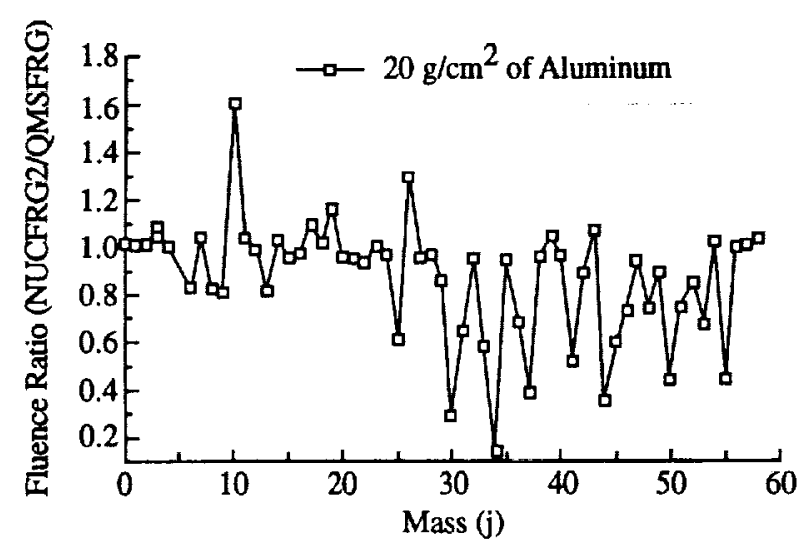

Fig. $4 c$. Same as $4 \mathrm{a}$ for $20 \mathrm{~g} / \mathrm{cm}^{2}$.

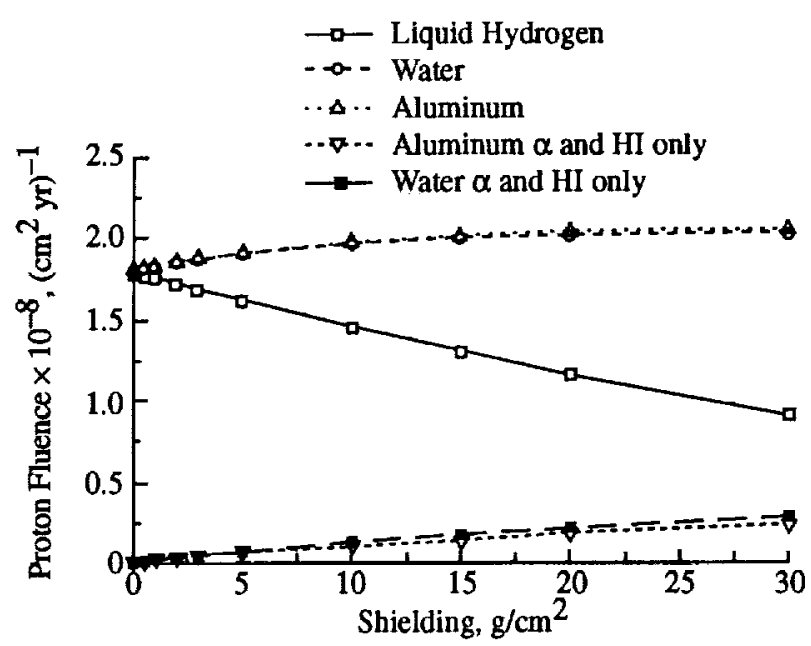

Fig. 5a. Calculations of proton fluence as a function of shielding depth for several shielding materials. Also shown are contributions to proton production from $\alpha$ and $\mathrm{HI}$. 


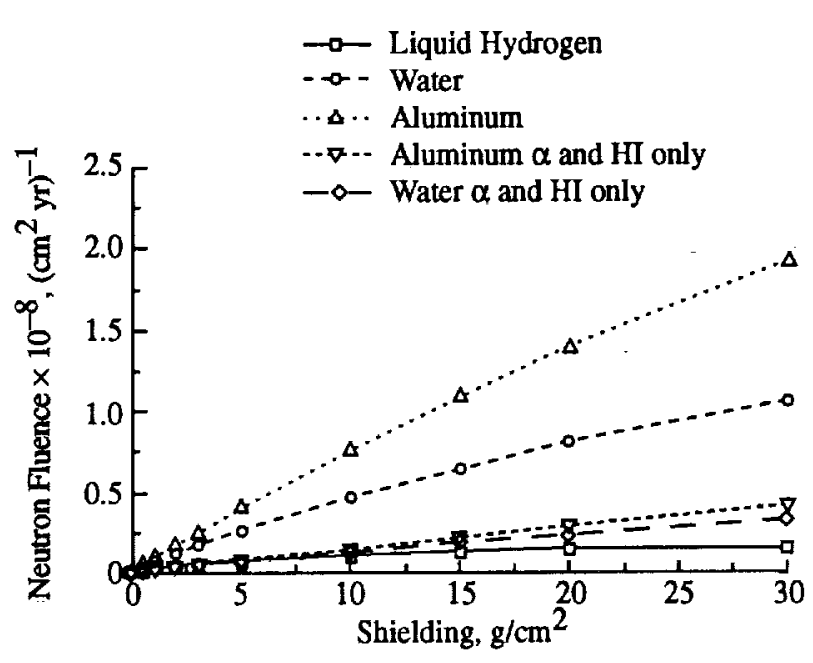

Fig. 5b. Same as Fig. 5a for neutrons.

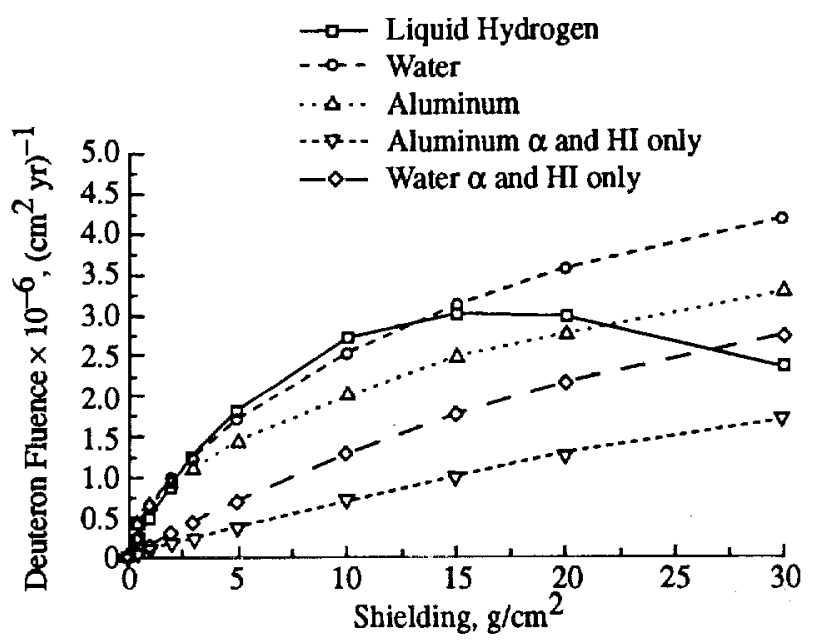

Fig. 5c. Same as Fig. 5a for deuterons. 


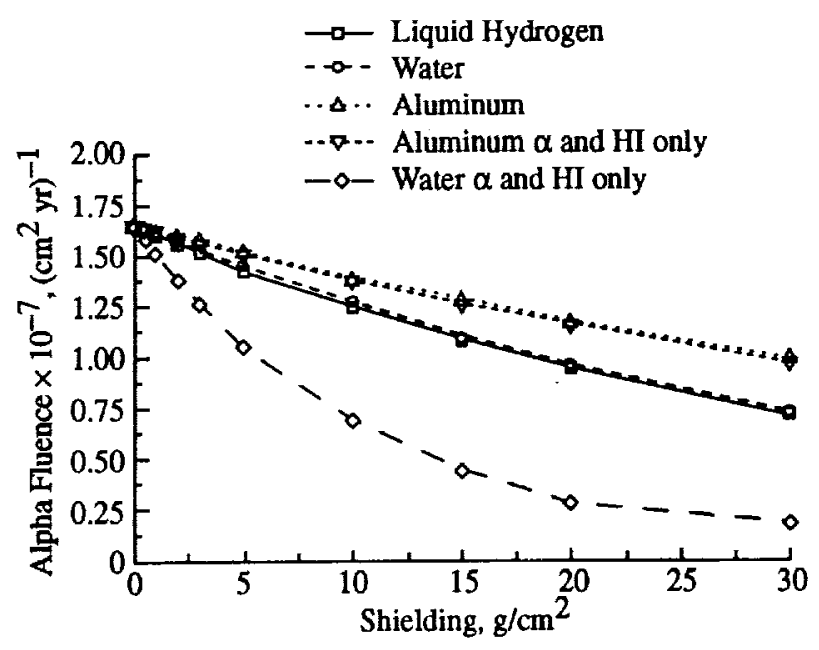

Fig. 5d. Same as Fig. 5a for alphas.

In Figure 6, we show the fraction of the total LET spectrum due to target fragments as a function of depth behind aluminium. The contribution from target fragments approaches $50 \%$ in the region from $50-200 \mathrm{keV} / \mu \mathrm{m}$ at the larger shielding depths. This region is dominated by low energy $\mathrm{H}$ and $\mathrm{He}$ isotopes and the spectrum of these particles is in near equilibrium as they are produced by the largely non-attenuated relativistic sources. The light production spectrum from $p, n, \alpha$ and $\mathrm{HI}$ induced reactions are important areas for future research based on these results and also due to the lack of thin target measurements. The cross sections for $p$ and $n$ induced reactions were discussed in Cucinotta et al. (1995a). The HZETRN code represents light particles production from projectile fragmentation using a velocity conserving interaction while a broad energy distribution is seen in HI experiments (Madey et al., 1985). The result of the QMSFRG model (Cucinotta, 1994b, Cucinotta et al., 1995b) for proton production from relativistic Ar beams and for neutron production from Ne beams on several targets are shown in Figures 7 . The model assumes two sources of neutrons from both projectile and target fragments, corresponding to direct knockout and cascades from the abrasion stage and evaporation neutrons from the ablation stage. Similar mechanisms for other light ions will contribute. These effects are currently under theoretical consideration and along with the inclusion of the meson contributions may represent the most significant improvement in the HZETRN database to be made in future work.

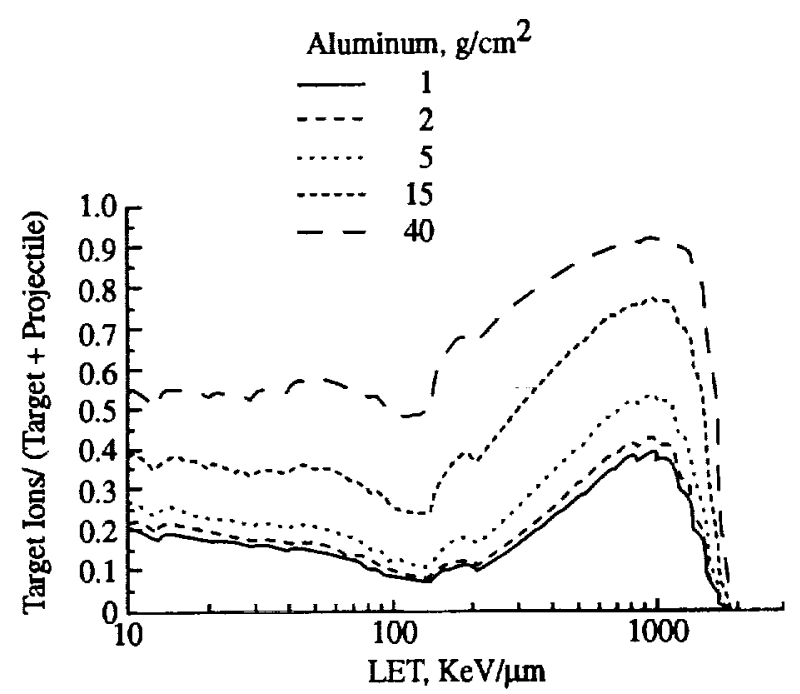

Fig. 6. Calculations using HZETRN code showing contribution of target fragments fluence to total fluence as a function of particle LET behind several depths of aluminum shielding. 


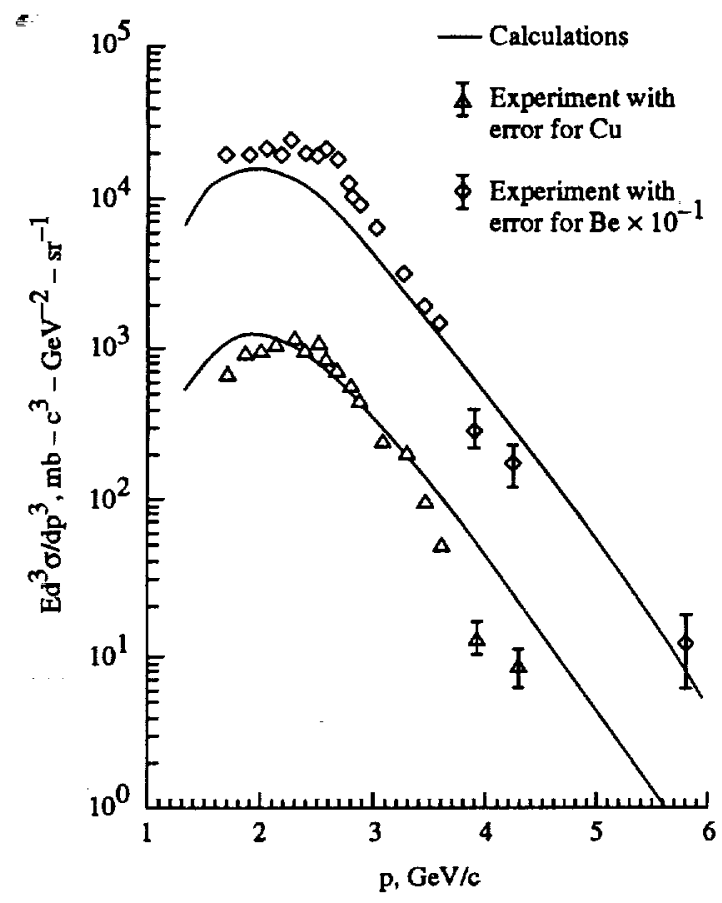

Fig. 7a. Comparisons of calculations with experiments for proton production at $5 \mathrm{deg}$ from Ar collisions on several target nuclei at $1.8 \mathrm{Ge} \mathrm{V} / \mathrm{u}$.

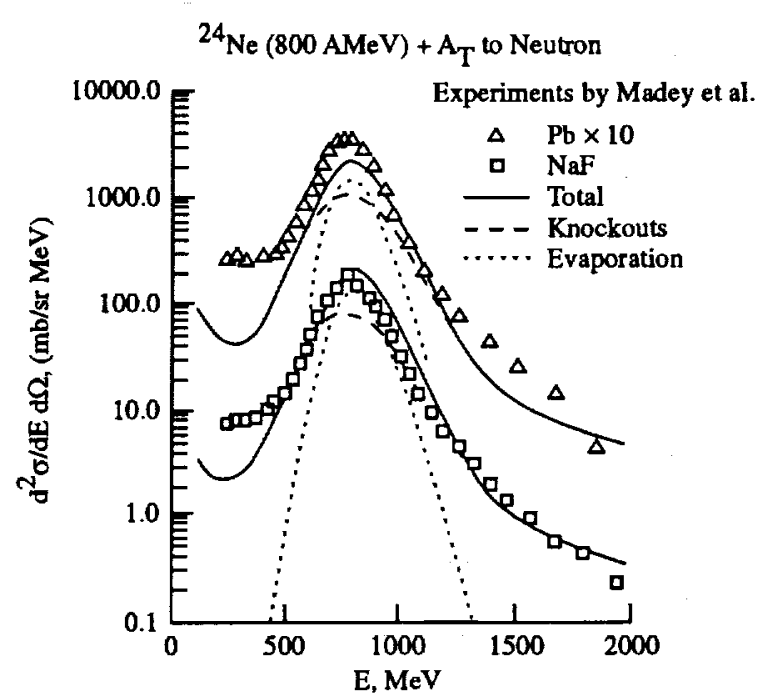

Fig. 7b. Comparisons of calculations with experiments for neutron productions at $0 \mathrm{deg}$ from $\mathrm{Ne}$ collisions on several target nuclei at $0.8 \mathrm{Ge} \mathrm{V/u}$.

\section{CONCLUSIONS}

An assessment of nuclear reaction data base requirements for GCR transport in structures and the atmosphere was given through comparisons using existing data bases and transport codes and with experimental data. A new fragmentation data base derived from a quantum mechanical formalism (QMSFRG) is shown to provide improved representation of the nuclear structure effects seen in experimental data in comparisons with the semi-phenomelogical NUCFRG2 model. A comparison of the secondary heavy ion fluences behind varying depths of aluminium shielding showed large differences for ions with reduced stability and good agreement between the two models for the more stable (and abundant) ions. The description of light particle production continues to be an important aspect for description of GCR transport in thick shielding including the atmosphere. The near equilibrium spectrum of low energy secondary hydrogen and helium isotopes is shown to rapidly contribute about $50 \%$ of the LET spectrum in the region of $50-200 \mathrm{keV} / \mu \mathrm{m}$. Future emphasis of data base development and measurements should adequately describe energy spectrum for these particles for both $p$ and $n$ induced reactions as well as $\alpha$ and heavy ion ones.

\section{REFERENCES}

Badhwar, G. D., J. U. Patel, F. A. Cucinotta, and J. W. Wilson, Measurements of the Secondary Particle Energy Spectra in the Space Shuttle, Radiat. Meas., 24, pp. 129-138, (1994).

Bowman, D., W. J. Swiatecki, and C. F. Tsang, Abrasion and Abalation of Heavy Ions, Lawrence Berkely Laboratory Report LBL-2908, Univ. of California, (1973).

Campi, X., and J. Hufner, Nuclear Spallation-Fragmentation Reactions Induced by High Energy Projectiles, Phys. Rev. C24, pp. 2199-2209, (1981).

Cucinotta, F. A., Calculations of Cosmic Ray Helium Transport in Shielding Materials, TP-3354, NASA, Washington, D. C., (1993).

Cucinotta, F. A., and R. D. Dubey, Alpha Cluster Model of ${ }^{12} \mathrm{C}\left({ }^{12} \mathrm{C}, 3 \alpha\right) \mathrm{X}$ at $2.1 \mathrm{~A} \mathrm{GeV}$, Phys. Rev. C50, pp. 974-984, (1994a).

Cucinotta, F. A., Forward Production of Protons From ${ }^{12} \mathrm{C}$ in Heavy Ion Collision, J. Phys. G., 20, pp. 1811-1821, (1994b). 
Cucinotta, F. A., L. W. Townsend, J. W. Wilson, J. L. Shinn, R. D. Dubey, and G. D. Budhwar, Light Ion Component of the Cosmic Rays: Nuclear Interactions and Transport Theory, Adv. Space Res. 17, pp. 77-86, (1995a).

Cucinotta, F. A., J. W. Wilson, and L. W. Townsend, Abrasion-Ablation Model For Neutron Production in Heavy Ion Reactions, TM-4656, NASA, Washington, D. C., (1995b).

Cucinotta, F. A., and J. W. Wilson, Study of Analytic Statistical Decay Model of the Decay of Light and Medium Mass Nuclei in Nuclear Fragmentation, TP in press, NASA, Washington, D. C., (1996).

Cummings, J. B., P. E. Haustein, T. J. Ruth and G. J. Virtes, Phys. Rev. C17, 1632, (1978).

Cummings, J. R., W. R. Binns, T. L. Garrand, M. H. Israel, J. Klarmann, E. C. Stone, and C. J. Waddington, Determination of the Cross Sections for the Production of Fragments from Relativistic Nucleus-Nucleus Interactions I. Measurements, Phys. Rev. C42, pp. 2508-2529 (1990).

Hufner, J., Schafer, and B. Schurmann, Abrasion-Ablation in Reactions Between Relativistic Heavy Ions, Phys. Rev. C12, pp. 1888-1898, (1975).

Kim, M. Y., J. W. Wilson, R. L. Kiefer, and S. A. Thibeault, Effects of Isotope Selection on Solution Convergence in HZE Transport, TP-3445, NASA, Washington, D. C., (1994).

Madey, R., J. Varga, A. R. Baldwin, B. D. Anderson, R. A. Cecil, G. Fai, P. C., Tandy and J. W. Watson, Inclusive Neutron Production Spectra at 0 deg From the Reactions $\mathrm{Pb}(\mathrm{Ne}, \mathrm{n}) \mathrm{X}$ and $\mathrm{NaF}$ (ne, n)X at 390 and $790 \mathrm{MeV}$ Per Nucleon, Phys. Rev. Lett., 55, pp. 1453-1456, (1985).

Rudstam, J., Systematics of Spallation Yields, Zeitschrift fur Naturforshung, 21a, pp. 1027-1041, (1966).

Shinn, J. L., S. John, R. K. Tripathi, J. W. Wilson, L. W. Townsend, and J. W. Norbury, Full Energy Dependent HZETRN (A Galatic Cosmic-Ray Transport Code), TP-3242, NASA, Washington, D. C., (1993).

Townsend, L. W., J. W. Wilson, J. W. Norbury, and F. A. Cucinotta, Comparisons of Abrasion-Ablation Models of Heavy Ion Fragmentation: Optical vs. Geometric, Phys. Rev. C34, pp. 1491-1494, (1986).

Westfall, G. D., T. J. M. Symons, D. E. Greiner, H. H. Heckman, P. J. Lindtrom, et al., Phys. Rev. Lett. 43, 1859, (1979).

Wilson, J. W., L. W. Townsend, and F. F. Badavi, A Semi-Empirical Nuclear Fragmentation Model, Nucl. Instrum. \& Methods Phys. Res. B18, pp. 225-231, (1987).

Wilson, J. W., L. W. Townsend, W. S. Schimmerling, G. S. Khandelwal, F. Khanet al., Transport Methods and Interactions for Space Radiations, NASA RP-1257, (1991).

Wilson, J. W., J. L. Shinn, L. W. Townsend, R. K. Tripathi, F. F. Badavi, S. Y. Chun, NUCFRG2: A Semi-Empirical Nuclear Fragmentation Model, Nucl. Instrum. \& Methods Phys. Res. B94, pp. 95-102, (1994).

Zeitlin, C., K. A. Frankel, L. Heilbronn, J. Miller, T. Borak, S. E. Rademacher, C. E. Stronachet al., Interactions of $1.09 \mathrm{~A} \mathrm{GeV}{ }^{56} \mathrm{Fe}$ Nuclei in Shielding and Tissue-Equivalent Materials, Presented at the 44th Annual Meeting of the Radiation Research Society, Chicago, Ill., (1996). 